\title{
Verletzungen, Behandlung und bleibende Schäden durch verschiedene Arten von Feuerwerkskörpern; Ergebnisse einer 10-jährigen multizentrischen retrospektiven Kohortenstudie
}

\author{
Daan T. Van Yperen ${ }^{a, b}$ Esther M.M. Van Lieshout ${ }^{a} \quad$ J. Niels Dijkshoorn ${ }^{b}$ \\ Cornelis H. Van der Vlies ${ }^{a, b}$ Michael H.J. Verhofstad ${ }^{a}$ \\ aTrauma Research Unit Department of Surgery, Erasmus MC, University Medical Center Rotterdam, Rotterdam, Niederlande; \\ ${ }^{b}$ Burn Center, Maasstad Hospital, Rotterdam, Niederlande
}

\author{
Schlüsselwörter \\ Verbrennungen · Epidemiologie · Feuerwerkskörper . \\ Schädigungsfolgen · Verletzung · Trauma
}

\begin{abstract}
Zusammenfassung
Ziele: Primäres Ziel der vorliegenden Studie war die Ermittlung der Zahl der Patienten, die während der vorangegangenen zehn Jahre in den Monaten Dezember und Januar mit Verletzungen durch Feuerwerkskörper ins Krankenhaus eingeliefert wurden; außerdem sollte der Zusammenhang zwischen der Art der Feuerwerkskörper, dem Verletzungsmuster, der Behandlung und den bleibenden Schäden beschrieben werden.
\end{abstract}

Methoden: Bei der vorliegenden Untersuchung handelt sich um eine multizentrische, retrospektive Beobachtungs-Fallserie. Die Patienten stammten aus zwei Krankenhäusern im Südwesten der Niederlande: einem Level-1-Traumazentrum und einer Spezialklinik für Brandverletzungen. Alle Patienten mit Verletzungen durch Feuerwerkskörper, die in den Jahren 2007 (Dezember) bis 2017 (Januar) zwischen dem 1. Dezember und dem 31. Januar behandelt worden waren, kamen für die Teilnahme in Frage. Primäres Zielkriterium war die Anzahl der Patienten mit einer durch Feuerwerkskörper verursachten Verletzung. Sekundäre Zielkriterien waren die Patientenmerkmale und Verletzungscharakteristika, die Behandlungsdetails und die Schädigungsfolgen (whole person impairment, WPI). Der Grad der Schädigungsfolgen in Prozent drückt den Grad der dauerhaften Beeinträchtigung eines Patienten infolge von Verletzungen durch Feuerwerkskörper aus.

Ergebnisse: Von 297 in Frage kommenden Patienten wurden 272 in die Studie eingeschlossen. Von 2007 bis 2017 wurden zwischen
21 und 40 Patienten behandelt, wobei kein eindeutiger Anstieg oder Rückgang der Patientenzahl und der Anzahl der Patienten pro Feuerwerkskörperart erkennbar war. Knallkörper verursachten vor allem Verletzungen der oberen Extremitäten ( $N=65 ; 68 \%)$, wohingegen Raketen ( $N=24 ; 41 \%)$ und Abschussrohre/-batterien ( $N=7$; 41\%) überwiegend die Kopf/Hals-Region betrafen. Leuchtkörper hatten hauptsächlich Verbrennungen ( $N=82 ; 68 \%)$ zur Folge und Knallkörper führten zu Weichteillazerationen ( $N=24 ; 25 \%)$, Frakturen $(N=16 ; 17 \%)$ sowie Amputationen $(N=14 ; 15 \%)$. Patienten mit Verletzungen durch Knallkörper und selbstgebaute Feuerwerkskörper wurden am häufigsten ins Krankenhaus eingeliefert ( $N=24$; $36 \%$ bzw. $N=12 ; 80 \%)$ und bei ihnen war der Anteil operativer Eingriffe am höchsten ( $N=22 ; 33 \%$ bzw. $N=7 ; 47 \%)$. Der in der vorliegenden Studie ermittelte WPI-Grad betrug zwischen 0 und 95\% und der Median lag bei 0\%. Bei 34 Patienten (14\%) führten die Verletzungen zu einem WPI-Grad von $\geq 1 \%$, meist als Folge einer Verwendung von Knallkörpern ( $N=18 ; 53 \%$ ).

Schlussfolgerung: In dieser Studie war kein Anstieg oder Rückgang der Anzahl der in zwei Spezialkliniken behandelten Patienten zu beobachten. Knallkörper und selbstgebaute Feuerwerkskörper können als die gefährlichsten angesehen werden, da sie mit den meisten Krankenhauseinweisungen und operativen Eingriffen verbunden sind und am häufigsten Verletzungen mit bleibenden Schäden verursachen.

(C) 2020 The Author(s) 


\section{Einleitung}

Auf der ganzen Welt werden Feuerwerkskörper eingesetzt, um der Freude über frohe Ereignisse, wie etwa Silvester, Ausdruck zu verleihen. Sie können jedoch trotz ihrer spektakulären visuellen und akustischen Effekte schwere Verletzungen wie Verbrennungen, Augenverletzungen und Amputationen verursachen [1-3]. Derartige Verletzungen können verheerende Auswirkungen und lebenslange Schäden zur Folge haben. Dadurch entstehen nicht nur zusätzliche medizinische Kosten aufgrund der physischen Verletzungen, sondern auch zusätzliche Kosten durch Vandalismus und Inanspruchnahme von Rettungsdiensten. Diese Probleme haben in den Niederlanden eine lang anhaltende öffentliche und politische Debatte über das Verbot von Feuerwerkskörpern ausgelöst.

Die meisten Verletzungen durch Feuerwerkskörper passieren in der Silvesternacht, da nur dann in den Niederlanden die Benutzung von Feuerwerkskörpern gestattet ist. Jährlich müssen zwischen 400 und 1000 Patienten im Krankenhaus behandelt werden. Seit 2012 ist diese Zahl auf etwa 400 Patienten in 2019-2020 zurückgegangen [4]. Meist handelte es sich um junge Männer vor allem mit Verbrennungen der oberen Extremitäten oder Augenverletzungen [1, 3, 5-7].

Das Dutch Safety Board - ein unabhängiges Beratungsgremium der Regierung - kam 2017 zu dem Schluss, dass die Silvesternacht das gefährlichste Ereignis in den Niederlanden darstellt, und empfahl, bestimmte Arten von Feuerwerkskörpern für den privaten Gebrauch zu verbieten, um die Sicherheit dieses Ereignisses zu erhöhen [8]. Das Gremium unterstrich zudem den Mangel an wissenschaftlichen Erkenntnissen über den Zusammenhang zwischen der Art der Feuerwerkskörper und den Verletzungen, die sie verursachen können. Solche Informationen sind sehr wichtig, denn sie können den politischen Entscheidungsträgern bei Entscheidungen, die die Verwendung von Feuerwerkskörpern sicherer machen, als Orientierungshilfe dienen.

Nach einem Bericht von Sandvall et al. war bei den Patienten, die in einem Level-1-Trauma-/Verbrennungszentrum behandelt wurden, der Anteil derjenigen, die wegen Verletzungen durch $\mathrm{Ku}$ gelbomben/Mörser operiert werden mussten, am größten [9]. Diese Art von Feuerwerkskörpern verursachte zudem die meisten Verletzungen, die bleibende Schäden zur Folge hatten. Billock et al. stellten in einer Untersuchung von 130.000 pädiatrischen Patienten fest, dass illegale/selbstgebaute Feuerwerkskörper den größten Anteil an Krankenhauseinweisungen ausmachten [5]. Solche Daten über die Auswirkungen bestimmter Arten von Feuerwerkskörpern fehlen in den Niederlanden.

Da Anzahl und Schweregrad der Verletzungen durch Feuerwerkskörper von Jahr zu Jahr stark schwanken [4], sind langfristige Daten erforderlich, die einen aussagekräftigen Überblick über die Folgen von Feuerwerkskörpern liefern. Daher bestand das primäre Ziel dieser Studie darin, die Zahl der Patienten zu ermitteln, die in den 10 Jahren zuvor während der Monate Dezember und Januar wegen Verletzungen durch Feuerwerkskörper in einem Krankenhaus behandelt wurden. Sekundäres Ziel war es, den Zusammenhang zwischen der Art der Feuerwerkskörper, dem Verletzungsmuster, der Behandlung und den bleibenden Schäden zu beschreiben.

\section{Material und Methoden}

\section{Design und Setting der Studie}

Bei der vorliegenden Untersuchung handelt es sich um eine retrospektive, multizentrische, Beobachtungs-Fallserie. Die potenziellen Teilnehmer wurden in zwei Schwerpunktkrankenhäusern im Südwesten der Niederlande, einem Level-1-Traumazentrum und einer Spezialklinik für Brandverletzungen, ausgewählt. Diese Studie wurde vom Erasmus MC Medical Research Ethics Committee (Rotterdam, Niederlande; Registrierungsnummer MEC2018-1254) als Studie mit Verzicht auf ein Ethikvotum (Exemption from Ethical Approval) eingestuft.

\section{Teilnehmer}

Alle Patienten (ohne Altersbegrenzung) mit Verletzungen durch Feuerwerkskörper, die in den Jahren 2007 (Dezember) bis 2017 (Januar) zwischen dem 1. Dezember und dem 31. Januar in einer der beiden teilnehmenden Kliniken behandelt worden waren, kamen für die Aufnahme in die Studie in Frage. Patienten, die innerhalb der ersten 24 Stunden aufgrund anderer Verletzungen verstarben oder deren Krankenakte in Bezug auf die Verletzungen unvollständig war, wurden von der Teilnahme ausgeschlossen. Von den teilnahmeberechtigten Patienten (oder deren Eltern bzw. Erziehungsberechtigten) musste eine informierte Einverständniserklärung eingeholt werden.

Zur Identifizierung potenzieller Teilnehmer wurden die Krankenakten anhand der Begriffe «Feuerwerkskörper», «Böller» und «Raketen» durchsucht. Es erfolgte ein Screening auf Eignung und die Teilnehmer wurden per E-Mail oder telefonisch um ihre Einwilligung nach Aufklärung gebeten. Zwei und vier Wochen später erhielten sie eine Erinnerung. Patienten, die die Teilnahme ablehnten, wurden ausgeschlossen. DTVY und JND untersuchten die Patienten auf ihre Eignung, holten die informierte Zustimmung ein und führten die Datenerhebung durch, indem sie Krankenakten der Patienten überprüften.

\section{Berechnung des Stichprobenumfangs}

Wegen des deskriptiven und nicht vergleichenden Studiendesigns war eine formale Berechnung der Stichprobengröße für diese Beobachtungsstudie nicht zielführend. Gemäß dem für die öffentliche Unfall- und Verletzungsprävention zuständigen niederländischen Institut für Verbrauchersicherheit wurden 2014 bis 2015 in den Niederlanden 574 Patienten mit Verletzungen durch Feuerwerkskörper behandelt [10]. Angesichts der 2-monatigen Einschlussfrist und der Beteiligung von zwei Schwerpunktkrankenhäusern für erweiterte Traumaversorgung und Brandverletzungen gingen die Schätzungen von etwa 200 Patienten in 10 Jahren aus. 
Tab. 1. Definition der verschiedenen Arten von Feuerwerkskörpern

\begin{tabular}{|c|c|c|}
\hline Art der Feuerwerkskörper & Definition & Beispiele \\
\hline Knallkörper & $\begin{array}{l}\text { Feuerwerkskörper, die explodieren und als Haupteffekt einen Knall verursachen. Visuelle } \\
\text { Effekte werden dabei kaum erzeugt. Oft als Böller bezeichnet. }\end{array}$ & $\begin{array}{l}\text { M-80s, Black Cats, } \\
\text { Kanonenschläge, Cobras }\end{array}$ \\
\hline Leuchtkörper & $\begin{array}{l}\text { Feuerwerkskörper, die vor allem einen visuellen Effekt, wie beispielsweise bunte } \\
\text { Flammen, erzeugen. }\end{array}$ & \\
\hline Raketen & $\begin{array}{l}\text { Röhrenförmige Geräte, die meist an einem Holzstab befestigt sind und durch eigenen } \\
\text { Antrieb in die Luft aufsteigen. }\end{array}$ & $\begin{array}{l}\text { Flaschenraketen, Sky } \\
\text { Rockets, Missiles }\end{array}$ \\
\hline Abschussrohre/-batterien & Geräte, die Kugelbomben in die Luft schießen. & Cakes, Mörser \\
\hline Sonstige & $\begin{array}{l}\text { Kleine Feuerwerkskörper, die in der Regel in der Hand gehalten oder auf dem Boden } \\
\text { verwendet werden. Es wird nur eine Flamme und keine Explosion erzeugt. }\end{array}$ & $\begin{array}{l}\text { Wunderkerzen, Wirbel, } \\
\text { Fontänen }\end{array}$ \\
\hline Karbid & $\begin{array}{l}\text { Calciumcarbid ist eine chemische Verbindung, die unter Bildung hochentzündlicher } \\
\text { Gase mit Wasser reagiert. Wird es entzündet, kommt es zu einer Explosion. Es ist ein } \\
\text { Brauch, mit Karbid Gegenstände aus alten Milchdosen zu schießen. }\end{array}$ & \\
\hline Selbstgebaute Feuerwerkskörper & $\begin{array}{l}\text { Selbst hergestellte Feuerwerkskörper, oder vorhandene Feuerwerkskörper, die in } \\
\text { irgendeiner Weise verändert wurden. Diese Art von Feuerwerkskörpern galt als illegal. }\end{array}$ & Rohrbomben \\
\hline
\end{tabular}

\section{Datenerhebung}

Primäres Zielkriterium war die Anzahl der Patienten mit einer durch Feuerwerkskörper verursachten Verletzung. Es wurde die Anzahl der Verletzungen, einschließlich Lokalisation und Art (z.B. Verbrennungen, Weichteilschäden oder Augenverletzungen), erfasst. Verbrennungen wurden als oberflächlich, mit partieller Zerstörung (dermal), mit vollständiger Zerstörung (subdermal) und gemischt (partiell und vollständig) klassifiziert. Augenverletzungen wurden nach der betroffenen anatomischen Region und Weichteilschäden als oberflächliche und tiefe Lazerationen klassifiziert. Amputationen von Fingern wurden als total oder partiell klassifiziert. Eine Amputation distal des Metacarpophalangealgelenks galt als partielle Amputation.

Sekundäre Zielkriterien waren die Patientenmerkmale und Verletzungscharakteristika, die Behandlungsdetails sowie der Grad der Schädigungsfolgen (whole person impairment, WPI) in Prozent. $\mathrm{Zu}$ den Patientenmerkmalen gehörten Alter und Geschlecht. Die Unfallinformationen beinhalteten die Rolle des Patienten (Benutzer oder umstehende Person), die Art des Feuerwerkskörpers (Knallkörper, Leuchtkörper, Kalziumkarbid oder selbstgebaut) und ob es sich um legale oder illegale Feuerwerkskörper handelte. Einzelheiten zur Definition der verschiedenen Arten von Feuerwerkskörpern sind in Tabelle 1 aufgeführt. Als Behandlungsmerkmale wurden die Einweisungsraten ins Krankenhaus und auf die Intensivstation sowie die Verweildauer erfasst. Außerdem wurden Anzahl und Art der operativen Eingriffe sowie die Notwendigkeit einer Rehabilitationsbehandlung (Physio-, Hand- und Ergotherapie) erfasst. Für jede Verletzung wurde der prozentuale WPI-Grad gemäß den Richtlinien der American Medical Association zur Bewertung bleibender Schäden erfasst [11]. Beispielsweise wurde die Amputation einer kompletten Hand mit einem WPI-Grad von $56 \%$ bewertet und ein erblindetes Auge mit $25 \%$. Bei Patienten mit mehr als einer Verletzung wurde entsprechend den Richtlinien ein kombinierter WPI-Prozentsatz ermittelt.

\section{Statistische Auswertung}

Die statistische Analyse erfolgte mit SPSS 25 (Statistical Package for the Social Sciences Version 25 (Chicago, IL, USA). Die Daten wurden gemäß den STROBE-Richtlinien (Strengthening the Reporting of Observational studies in Epidemiology) angegeben.

Kontinuierliche Daten wurden mit dem Shapiro-Wilk-Test auf Normalität getestet. Dabei zeigte sich, dass alle Daten von der Standardnormalverteilung abwichen. Fehlende Werte wurden nicht mittels Imputation ersetzt.

Zur Darstellung der Daten für das Gesamtkollektiv sowie für die wichtigsten Arten von Feuerwerkskörpern erfolgten deskriptive Analysen. Kontinuierliche Daten wurden als Medianwerte und Quartile angegeben. Kategoriale Daten sind als Anzahl und Häufigkeiten dargestellt. Ein statistischer Vergleich zwischen den verschiedenen Arten von Feuerwerkskörpern erfolgte nicht.

\section{Ergebnisse}

\section{Teilnehmer}

Von den 297 Patienten, die die Eignungskriterien erfüllten, wurden 25 Patienten ausgeschlossen, weil sie eine Teilnahme ablehnten. Insgesamt wurden 272 Patienten in diese Studie aufgenommen (Abb. 1). Die Dauer der klinischen Nachbeobachtung betrug im Median 13 Tage $\left(\mathrm{P}_{25}-\mathrm{P}_{75} 3-87\right)$.

Abbildung 2 zeigt die Zahl der jährlich behandelten Patienten. In der Abbildung sind Informationen zum Gesamtkollektiv und zu den verschiedenen Arten von Feuerwerkskörpern dargestellt. Von 2007 bis 2017 wurden zwischen 21 und 40 Patienten pro Jahr behandelt. Die Gesamtzahl der behandelten Patienten stieg von 24 im Jahr 2007 auf 40 Patienten im Jahr 2014 und ging danach auf 22 Patienten im Jahr 2017 zurück. Der größte Teil der Verletzungen in den Jahren 2010 bis 2014 war durch Knallkörper verursacht. In diesem Zeitraum hatten zwischen 20\% $(N=8)$ und $42 \%(N=13)$ der Patienten Verletzungen, die durch Knallkörper bedingt waren. Ab 2014 kam es zu einem Rückgang dieser Zahl 


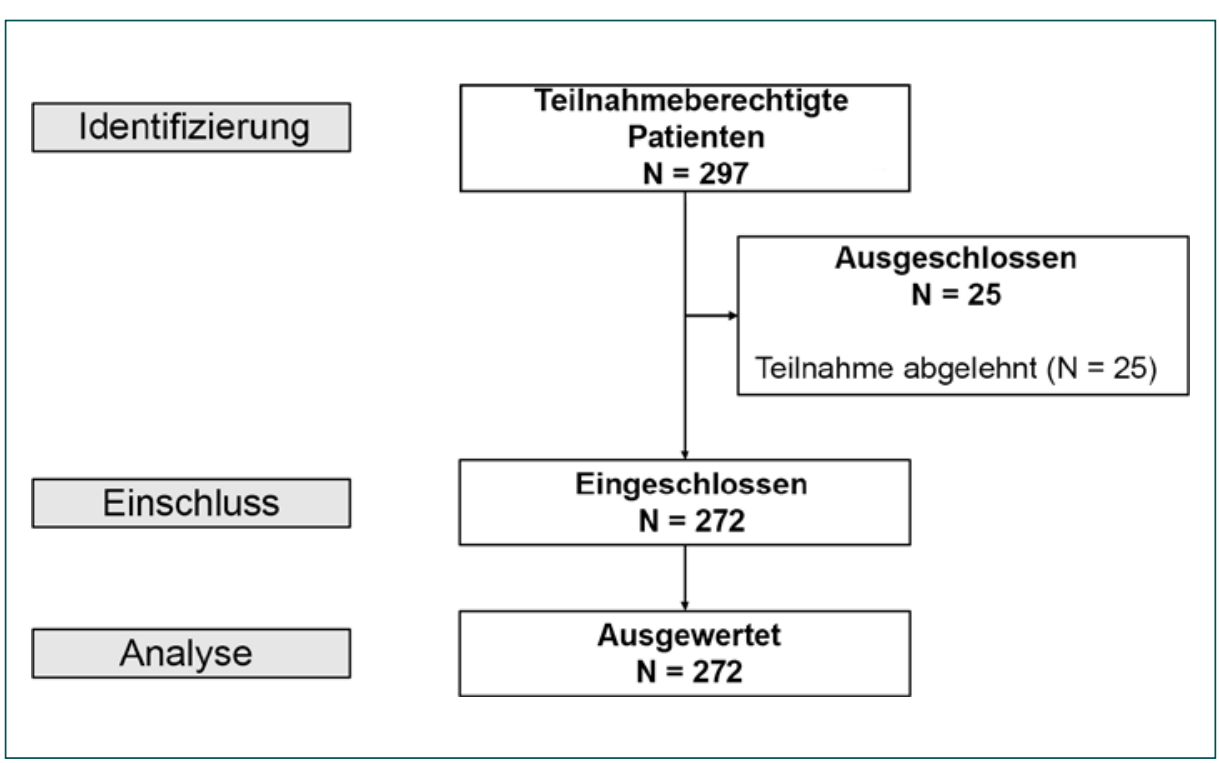

Abb. 1. Ablaufdiagramm der Studie.

Abb. 2. Anzahl der pro Jahr behandelten Patienten. Anzahl der Patienten mit Verletzungen durch Feuerwerkskörper, die jährlich im Dezember und Januar behandelt wurden, nach Art der Feuerwerkskörper.

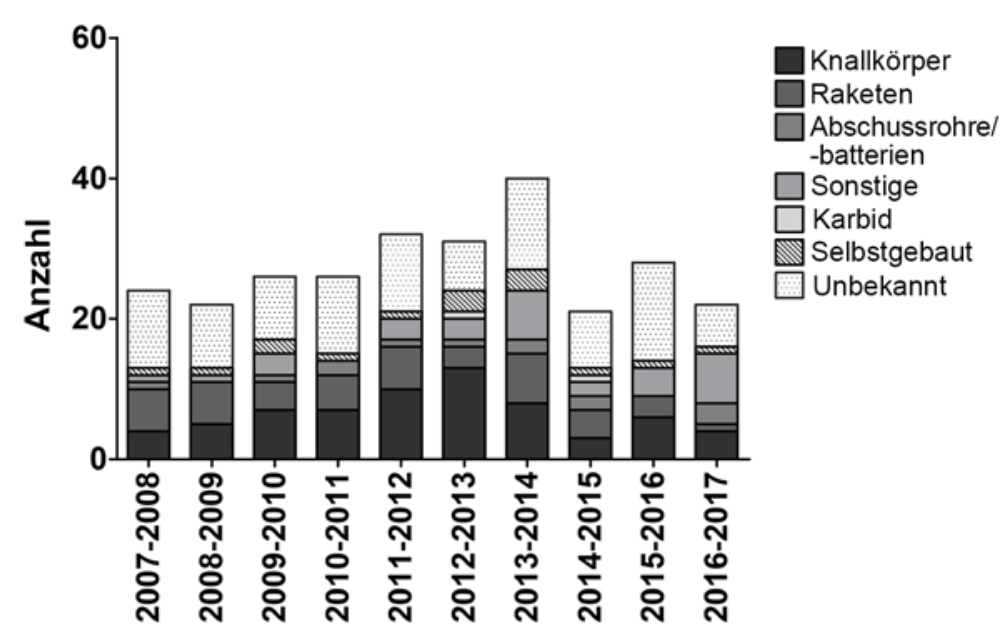

Jahr

auf ein ähnliches Niveau wie bei den anderen Feuerwerkskörperarten. Die Zahl der durch Raketen verletzten Patienten fiel in den letzten vier Jahren von sieben Patienten im Jahr 2014 auf einen Patienten im Jahr 2017. In Hinblick auf die Gesamtzahl der behandelten Patienten und die Anzahl der Patienten pro Feuerwerkskörperart war kein eindeutiger Anstieg oder Rückgang zu beobachten.

\section{Patienten-Charakteristika}

Tabelle 2 enthält Angaben zu den Merkmalen der Patienten und der Feuerwerkskörper. Der Altersmedian des Gesamtkollektivs lag bei 18 Jahren $\left(\mathrm{P}_{25}-\mathrm{P}_{75} 13-30\right)$. Insgesamt waren unter den $\mathrm{Pa}$ tienten 114 Kinder (42\%) unter 16 Jahren. Die Patienten waren überwiegend männlich $(N=227 ; 84 \%)$, und bei fast der Hälfte aller Patienten $(N=87 ; 40 \%)$ handelte es sich um umstehende
Personen. Mehr als die Hälfte der Patienten $(N=50 ; 60 \%)$ wurde durch legale Feuerwerkskörper verletzt.

Die meisten Verletzungen $(N=89 ; 51 \%)$ wurden durch Leuchtkörper verursacht, insbesondere durch Raketen $(N=45 ; 50 \%)$, gefolgt von Knallkörpern $(N=67 ; 39 \%)$. Bei 99 Patienten (25\%) war die Art des Feuerwerkskörpers unbekannt. Der Altersmedian variierte je nach Art der Feuerwerkskörper erheblich und reichte von 13 Jahren in der Gruppe «Sonstige» bis zu 29 Jahren in der Gruppe «Abschussrohre/-batterien». Abgesehen von der Untergruppe «Raketen» $(N=29 ; 64 \%)$ lag der Anteil der Männer in allen Untergruppen bei mehr als 80\%. Bei den Raketen war der Anteil verletzter umstehender Personen am höchsten $(N=27$; 77\%); in allen anderen Gruppen betrug ihr Anteil weniger als $39 \%$. 


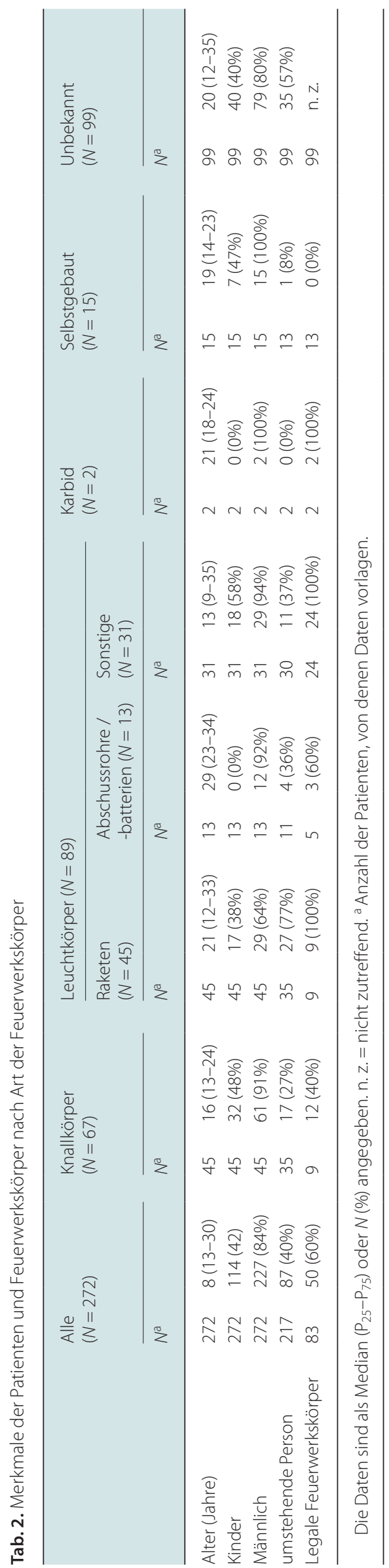

\section{Verletzungsmerkmale}

Insgesamt wurden 395 Verletzungen bei 272 Patienten berichtet. Die meisten Verletzungen betrafen die oberen Extremitäten $(N=$ $177 ; 45 \%)$, gefolgt von der Kopf/Hals-Region $(N=101 ; 26 \%)$ und Augen $(N=49 ; 12 \%$; Tabelle 3$)$. Knallkörper verursachten vor allem Verletzungen der oberen Extremitäten $(N=65 ; 68 \%)$, wohingegen Raketen $(N=24 ; 41 \%)$ und Abschussrohre/-batterien $(N=$ 7; 41\%) vorwiegend Verletzungen der Kopf/Hals-Region verursachten.

Die häufigsten Verletzungen waren Verbrennungen $(N=208$; $53 \%)$, und sie waren meist mit partieller Zerstörung verbunden $(N=162 ; 78 \%)$. Die zweithäufigste Verletzungsart waren Weichteillazerationen $(N=67 ; 17 \%)$ - größtenteils von oberflächlicher Natur $(N=48 ; 73 \%)$ - und am dritthäufigsten waren Augenverletzungen $(N=49 ; 12 \%)$. Sechs (6) Patienten hatten eine bilaterale Augenverletzung. Bei einem Kind und fünf Erwachsenen war ein Auge komplett zerstört und sie galten als im juristischen Sinne blind. Bei 31 Verletzungen (8\%) handelte es sich um Frakturen, deren häufigste Lokalisation Finger $(N=19 ; 61 \%)$, Hand $(N=7$; $23 \%)$ und Gesicht $(N=6 ; 19 \%)$ waren. Ferner traten 19 (5\%) Amputationen auf, die alle die Hand oder Finger betrafen. Zwei Hände, 32 Finger (davon 29 partiell) und vier Daumen (davon zwei partiell) wurden amputiert.

Leuchtkörper verursachten überwiegend Brandverletzungen $(N=82 ; 68 \%)$, von denen 60 (73\%) mit partieller Zerstörung einhergingen. Knallkörper führten meist zu Weichteillazerationen $(N=24 ; 25 \%)$, Frakturen $(N=16 ; 17 \%)$ und Amputationen $(N=$ 14; 15\%). Selbstgebaute Feuerwerkskörper verursachten hauptsächlich Verbrennungen $(N=18 ; 58 \%)$. Augenverletzungen waren vor allem durch Raketen $(N=12 ; 24 \%)$ und Knallkörper $(N=8$; $16 \%)$ bedingt.

\section{Therapie}

Insgesamt wurden 79 Patienten (29\%) zur Behandlung ins Krankenhaus eingeliefert, und 10 Patienten (4\%) wurden auf die Intensivstation aufgenommen (Tabelle 4). Die mediane Verweildauer betrug 4 Tage $\left(\mathrm{P}_{25}-\mathrm{P}_{75} 2-9\right)$. Einundfünfzig (51) Patienten (19\%) wurden operiert, davon 22 (42\%) zweimal oder mehrfach, im Höchstfall bis zu zehn Mal. Darüber hinaus erhielten 57 Patienten (22\%) Patienten eine professionelle Rehabilitationsbehandlung, davon die meisten eine Physiotherapie $(N=29 ; 51 \%)$ oder eine HandtherapIe $(N=35 ; 61 \%)$.

Am häufigsten wurden Patienten mit Verletzungen durch Karbid und selbstgebaute Feuerwerkskörper ins Krankenhaus eingeliefert $(N=2 ; 100 \%$ und $N=12 ; 80 \%)$, gefolgt von Patienten mit Verletzungen durch Knallkörper $(N=24 ; 36 \%)$. Die längste Verweildauer von 9 Tagen $\left(\mathrm{P}_{25}-\mathrm{P}_{75} 3-18\right)$ hatten die Patienten der Untergruppe «Sonstige». Selbstgebaute Feuerwerkskörper $(N=7 ; 47 \%)$ und Knallkörper $(N=22 ; 33 \%)$ machten den größten Anteil an Verletzungen aus, die eine Operation erforderten.

\section{Schädigungsfolgen}

In der vorliegenden Studien wurden keine Todesfälle beobachtet. Der Grad der Schädigungsfolgen (whole person impairment, 


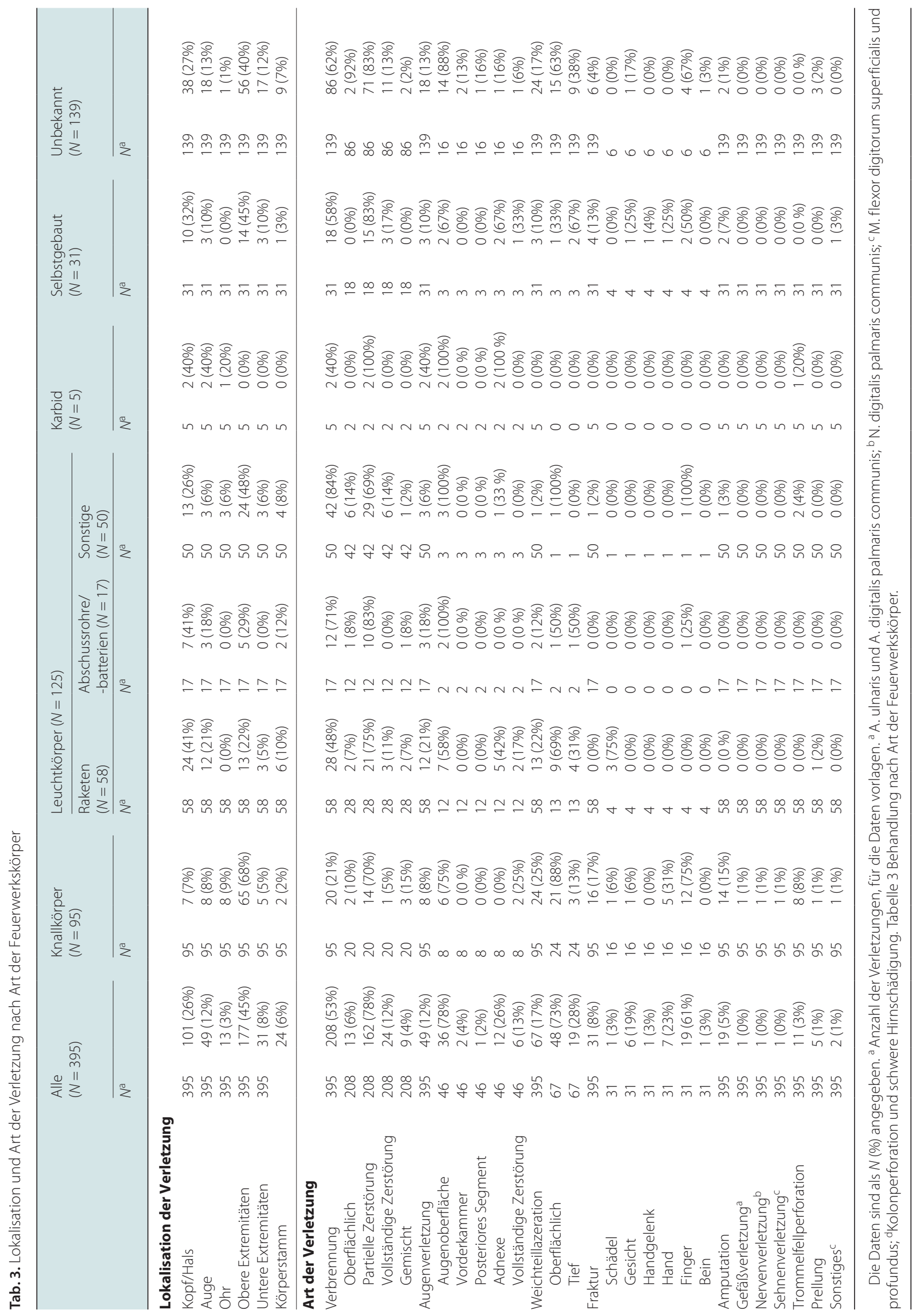




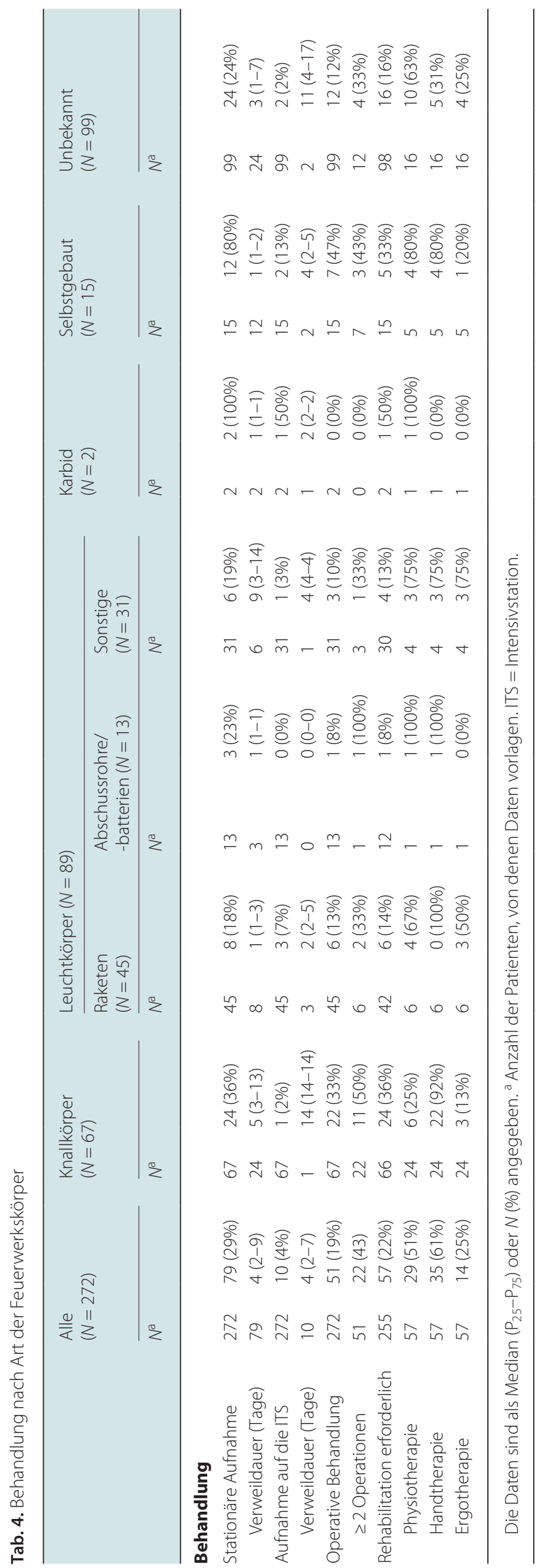

WPI) betrug in dieser Studie zwischen 0 und 95\%. Bei 34 Patienten (14\%) hatten die Verletzungen einen WPI-Grad von $\geq 1 \%$ zur Folge; von diesen hatten 18 Patienten (53\%) Knallkörper benutzt, 4 (12\%) hatten selbstgebaute Feuerwerkskörper verwendet, 4 (12\%) hatten Raketen benutzt, 2 (6\%) «sonstige» Feuerwerkskörper und bei 6 Patienten (18\%) war die Art der verwendeten Feuerwerkskörper nicht bekannt. Bei 217 Patienten (84\%) führten die Verletzungen nicht zu bleibenden Schäden, und bei 21 Patienten (8\%) konnte der WPI-Grad wegen fehlender Daten nicht ermittelt werden. In allen Gruppen betrug der WPI-Grad im Median 0\% $\left(\mathrm{P}_{25}-\mathrm{P}_{75} 0-0\right)$.

Ein Kind erlitt ein Schädel-Hirn-Trauma durch Splitter eines illegalen Böllers, das zu einem WPI-Grad von 95\% führte. Ein anderes Kind verlor durch einen Böller eine Hand und ein Erwachsener verlor eine Hand durch unbekannte Feuerwerkskörper, was in beiden Fällen einen WPI-Grad von 56\% zur Folge hatte. Siebzehn (17) Patienten mit Einfach- oder Mehrfachamputation der oberen Extremitäten hatten einen WPI-Grad zwischen 2\% bei Amputation einer einzelnen Fingerkuppe und 46\% bei einer ausgedehnten Handverletzung mit Amputation mehrerer Finger. Bei 14 von diesen Patienten waren die Verletzungen durch Knallkörper bedingt. Sechs (6) Patienten verloren ein Auge und erblindeten, was einen WPI-Grad von $25 \%$ zur Folge hatte. Ursache für eine Erblindung waren Knallkörper $(N=2)$, Raketen $(N=2)$, selbstgebaute Feuerwerkskörper $(N=1)$ sowie unbekannte Feuerwerkskörper $(N=1)$.

\section{Diskussion}

Die vorliegende Studie untersuchte retrospektiv Patienten, die über einen Zeitraum von 10 Jahren wegen feuerwerkskörperbedingter Verletzungen in zwei spezialisierten Kliniken behandelt wurden. Das wichtigste Ergebnis war, dass die Zahl der jährlich behandelten Patienten zwischen 21 und 40 schwankte und über die vergangenen zehn Jahre weder eine Zunahme noch ein Rückgang zu beobachten war. Ferner führten Verletzungen durch Knallkörper und selbstgebaute Feuerwerkskörper am häufigsten $\mathrm{zu}$ Krankenhauseinweisungen und operativen Behandlungen, und sie waren am häufigsten mit bleibenden Schäden verbunden. Die Zahl der jährlich behandelten Patienten zeigte in den vergangenen Jahren weder einen Anstieg noch einen Rückgang, was den Angaben in der Literatur der letzten zwanzig Jahre entspricht [1, 2, 5, 12-16]. Landesweit sank die absolute Zahl der behandelten Patienten in den Niederlanden von 700 Patienten im Jahr 2014 auf 385 im Jahr 2020 [4]. Seit Jahren versuchen die politischen Entscheidungsträger mithilfe von Informations- und Aufklärungskampagnen, die Zahl der Verletzungen durch Feuerwerkskörper zu senken. Homaie Rad et al. unterstrichen in ihrer kürzlich veröffentlichten Vorher-Nachher-Studie die Wirksamkeit solcher Interventionen in Hinblick auf die Verringerung der behinderungsbereinigten Lebensjahre und der Krankheitslast [17]. In den oben genannten Studien wurden die Effekte derartiger Kampagnen jedoch noch nicht festgestellt. Möglicherweise waren diese Kampagnen nur bei Patienten mit leichten Verletzungen, die kei- 
ne Krankenhausbehandlung erforderten, wirksam. Die präventiven Maßnahmen zeigten weniger Wirksamkeit als erwartet, wohingegen sich restriktive rechtliche Vorschriften als wirksam erwiesen und die Zahl der Verletzungen Feuerwerkskörper verringerten [18-20].

Nur zwei frühere Studien beschrieben die Art der Feuerwerkskörper und den Zusammenhang mit Verletzungen, Behandlung und Outcome. Ein Vergleich dieser Studien war wegen der unterschiedlichen Gruppierung der Feuerwerkskörperarten schwierig. In einer US-amerikanischen Studie wurden mehr als 130000 pädiatrische Patienten untersucht und es zeigte sich, dass illegale/ selbstgebaute Feuerwerkskörper für den größten Anteil an Krankenhauseinweisungen verantwortlich waren [5]. Allerdings wurden in dieser Studie illegale Feuerwerkskörper in einer Gruppe mit selbstgebauten Feuerwerkskörpern zusammengefasst, während in der vorliegenden Studie legale und illegale Feuerwerkskörper gemeinsam zur Gruppe der Knallkörper zählten. Sandvall et al. schlossen in ihre Studie Patienten eines Level-1-Trauma-/ Verbrennungszentrums ein, und stellten fest, dass in der Gruppe Kugelbomben/Mörser der Anteil an Operationen am größten war, gefolgt von selbstgebauten Feuerwerkskörpern. Diese beiden Gruppen waren auch für den größten Teil an Augen- und Handverletzungen verantwortlich, die bleibende Schäden zur Folge hatten. Beide oben genannten Studien weisen Ähnlichkeiten mit unserer Studie auf und verdeutlichen die Gefahren von selbstgebauten und (illegalen) Knallkörpern. Selbstgebaute Feuerwerkskörper sind definitionsgemäß illegal und dürfen daher privat nicht verwendet werden, allerdings sind nicht alle Knallkörper illegal. Die vorliegende Studie traf keine Unterscheidung zwischen legalen und illegalen Feuerwerkskörpern, da dies lediglich bei weniger als einem Drittel der Patienten erfasst wurde.

Diese Studie zeigte zudem, dass insbesondere Raketen für die Umstehenden gefährlich waren. Der Anteil der Umstehenden, die von Raketen betroffen waren, lag viel höher als bei anderen Arten von Feuerwerkskörpern. Bei Raketen besteht nicht nur für den Bediener eine Gefahr, sondern auch für die umstehenden $\mathrm{Zu}$ schauer. Dies wurde auch schon früher berichtet [9]. Raketen werden oft aus leeren Flaschen abgefeuert und können leicht umkippen oder abgelenkt werden und Umstehende treffen. Das Dutch Safety Board empfahl 2017, die private Verwendung von Raketen und Knallkörpern zu verbieten, da diese beiden Arten von Feuerwerkskörpern die allgemeine Sicherheit in der Silvesternacht am stärksten gefährdeten [8]. Im Jahr 2020 folgte die niederländische Regierung diesem Rat und beschloss ein Gesetz, das den privaten Gebrauch von Knallkörpern und Feuerwerksraketen verbietet. Die Auswirkungen dieser Maßnahme müssen sich erst noch zeigen.

Unseres Wissens gehört diese Studie zu den wenigen, in denen eine Berechnung der bleibenden Schäden durch feuerwerkskörperbedingte Verletzungen erfolgte. Nur Sandvall et al. führten eine solche durch und ermittelten einen WPI-Grad zwischen $1 \%$ und $77 \%$, was mit der aktuellen Studie vergleichbar ist [9]. In der vorliegenden Studie wurde ein WPI zwischen 0 und 95\% festgestellt. Diese große Spannbreite ist vor allem durch die geringe
Zahl von Patienten bedingt, bei denen bleibende Schäden objektiv nachgewiesen wurden, sowie auf die Vielzahl der in jedem Jahr auftretenden Verletzungsmuster.

Die meisten Verletzungen, die bleibende Schäden zur Folge hatten, wurden durch Knallkörper und selbstgebaute Feuerwerkskörper verursacht. Vierzehn (14) von 18 Patienten mit bleibenden Schäden infolge von Knallkörpern erlitten Amputationen. Bei dieser Art von Feuerwerkskörpern kann es vor allem durch die Explosion leicht zu Amputationen kommen, die unausweichlich bleibende Schäden zur Folge haben. Demgegenüber erzeugen Leuchtkörper hauptsächlich Lichtblitze und keine Explosion und führen daher meist zu oberflächlichen Verbrennungen von geringer Größe [2, 16]. Zwar weist vermutlich ein Großteil der Patienten dieser Studie Narben auf, doch spiegelt sich dies nicht im prozentualen Grad der Schädigungsfolgen wider, da eine Beeinträchtigung durch Narben erst dann entsteht, wenn 10\% der gesamten Körperoberfläche betroffen sind. Daher ist die Wahrscheinlichkeit für bleibende Schäden bei Leuchtkörpern sehr viel geringer als bei Knallkörpern. Dennoch zeigt die große Spanne der in dieser Studie festgestellten prozentualen WPI-Grade die potenziellen und verheerenden Auswirkungen von Feuerwerkskörpern. Ob diese Schäden auch funktionelle Beeinträchtigungen zur Folge haben, ist nicht umfassend untersucht worden. In früheren Forschungsarbeiten wurde eine begrenzte Einschränkung der Lebensqualität und des funktionellen Outcomes ein Jahr nach dem berichteten Trauma beobachtet [3].

\section{Stärken und Einschränkungen}

Eine Stärke der vorliegenden Studie besteht darin, dass sie einen Langzeitüberblick über Patienten gibt, die in Schwerpunktkrankenhäusern behandelt wurden, die häufig Patienten mit schweren Verletzungen durch Feuerwerkskörper versorgen. Diese Informationen liefern Erkenntnisse über die Zahl der in den vergangenen 10 Jahren behandelten Patienten und ihr Outcome nach mehreren Jahren. Außerdem gibt die Studie näheren Aufschluss über die Verletzungen durch Feuerwerkskörper und sie beschreibt den Zusammenhang zwischen der Art der Feuerwerkskörper und den Verletzungen, der Behandlung und dem Ausmaß der bleibenden Schäden. Dies wird den politischen Entscheidungsträgern helfen, die beste Strategie zur Verhütung von Verletzungen durch Feuerwerkskörper zu entwickeln.

Eine Einschränkung in dieser Studie könnte sein, dass nur zwei Spezialkliniken für die Teilnahme an dieser Studie ausgewählt wurden. In dieser Studie sollte das Hauptaugenmerk auf schwer verletzte Patienten gelegt werden, deren Behandlung mit höherer Wahrscheinlichkeit in einem spezialisierten Krankenhaus erfolgt. Patienten mit schweren Augenverletzungen wurden jedoch häufig sofort in eine nahe gelegene spezialisierte Augenklinik, die auch nationales Referenzzentrum ist, gebracht und deshalb nicht in diese Studie eingeschlossen. Daher waren Patienten mit schweren Augenverletzungen wahrscheinlich unterrepräsentiert. Die Daten der in dieser spezialisierten Augenklinik behandelten Patienten wurden kürzlich veröffentlicht [21]. Wegen des retrospektiven Studiendesigns waren zudem manche Daten wie 
beispielsweise die Art der Feuerwerkskörper nicht in den Krankenakten der Patienten erfasst worden. Daher ist bei Aussagen über die Kausalität Vorsicht geboten. Ein weiterer potenzieller Schwachpunkt dieser Studie besteht darin, dass die funktionelle Beeinträchtigung allein auf Grundlage von Daten aus den Krankenhausakten errechnet wurde. Es ist klar, dass Amputationen und die Enukleation eines Auges mit dauerhaften Beeinträchtigungen verbunden sind. Wahrscheinlich wäre der Grad der Schädigungsfolgen - auch bei denjenigen, die aktuell einen WPIGrad von $0 \%$ hatten - nach einer gezielten körperlichen Untersuchung zum Zweck dieser Studie jedoch höher ausgefallen. Es kann sehr gut sein, dass die Berechnung auf Grundlage kurzfristiger Krankenhausaufzeichnungen zu zu niedrigen Schätzwerten geführt hat.

\section{Schlussfolgerung}

Mit der vorliegenden Studie sollte primär die Zahl der Patienten untersucht werden, die in den vorangegangenen zehn Jahren während der Monate Dezember und Januar mit Verletzungen durch Feuerwerkskörper im Krankenhaus behandelt wurden. Außerdem sollte der Zusammenhang zwischen der Art der Feuerwerkskörper, dem Verletzungsmuster, der Behandlung und den bleibenden Schäden beschrieben werden. Im Studienzeitraum war kein Anstieg oder Rückgang der Zahl der behandelten Patienten zu beobachten. Die Studie ergab, dass Knallkörper und selbstgebaute Feuerwerkskörper als am gefährlichsten angesehen werden können, da sie mit den höchsten Raten an Krankenhauseinweisungen, operativen Eingriffen und verletzungsbedingten bleibenden Schäden assoziiert sind. Insbesondere Raketen waren für Umstehende gefährlich. Zwar führten die meisten Verletzungen nicht zu bleibenden Schäden, doch hatten sie bei einigen $\mathrm{Pa}-$ tienten lebenslange und schwere Behinderungen zur Folge.
Die Ergebnisse dieser Studie müssen in die öffentliche und politische Debatte einfließen und die politischen Entscheidungsträger weltweit dabei unterstützen, die Probleme durch Feuerwerkskörper zu lösen. Zukünftige Untersuchungen sollten die Entwicklung sichererer Alternativen für Feuerwerkskörper wie beispielsweise Laser, Drohnen und andere nicht feuererzeugende Apparaturen in den Blick nehmen.

\section{Verfügbarkeit der Daten und Materialien}

Die in dieser Arbeit analysierten anonymisierten Datensätze sind auf begründete Anfrage durch einen qualifizierten Prüfer vom Korrespondenzautor erhältlich.

\section{Genehmigung durch die Ethikkommission und Einwilli- gung in die Teilnahme}

Diese Studie wurde vom Erasmus MC Medical Research Ethics Committee (Rotterdam, Niederlande; Registrierungsnummer MEC-2018-1254) als Studie mit Verzicht auf ein Ethikvotum (Exemption from Ethical Approval) eingestuft. Da nur retrospektive Beobachtungsdaten erhoben wurden, war eine schriftliche Einwilligung nach Aufklärung nicht erforderlich.

\section{Disclosure Statement}

Die Autoren erklären, dass keine Interessenkonflikte bestehen.

\section{Lizenzangabe}

Van Yperen DT, Van Lieshout EMM, Dijkshoorn JN, Van der Vlies CH, Verhofstad MHJ: Injuries, treatment, and impairment caused by different types of fireworks; results of a 10 year multicenter retrospective cohort study. Scand J Trauma Resusc Emerg Med. 2021;29(1):11. (DOI: 10.1186/ s13049-020-00811-z). ${ }^{\odot} 2020$ The Author(s). (Übersetzung; Abbreviations, Acknowledgements, Author's Contribution, Funding, Consent for Publication und Publisher's Note gekürzt), lizensiert unter CC BY 4.0 (https://creativecommons.org/licenses/by/4.0/deed.de).

\section{Literatur}

Die Literatur ist unter www.karger.com/doi/10.1159/000520337 abrufbar. 